\title{
Calcitriol, Parathyroid Hormone, and Accumulation of Aluminum in Bone in Dogs with Renal Failure
}

Hartmut H. Malluche, Marie-Claude Faugere, Robert M. Friedler, Clifford Matthews, and Paolo Fanti

Division of Nephrology, Bone and Mineral Metabolism, Department of Medicine,

University of Kentucky, Lexington, Kentucky 40536-0084

\begin{abstract}
Accumulation of aluminum in bone is a frequent finding in patients requiring chronic dialysis and is associated with considerable morbidity and/or mortality. Until now, evidence seemed to point to relatively low circulating levels of parathyroid hormone as a contributing factor, but because levels of parathyroid hormone and calcitriol are interrelated, calcitriol might be also involved. In this study we employed an animal model to evaluate the single and combined effects of parathyroid hormone and calcitriol on bone aluminum accumulation. The results show significantly less aluminum accumulation in calcitriol-replete dogs independent of the presence or absence of parathyroid hormone. These results indicate that low levels of calcitriol may play a role in the development of aluminum related bone disease. Further studies are needed to demonstrate whether administration of calcitriol in patients with renal insufficiency will prevent development of aluminum-related bone disease.
\end{abstract}

\section{Introduction}

The accumulation of aluminum in bone is a frequent finding in patients with renal failure who require chronic dialysis (1-4). It may be associated with considerable morbidity, if not mortality (4). One of the puzzling aspects is the rather striking variance in the occurrence of aluminum-related bone disease and degrees of severity among patients afflicted with the disease. This has sparked research efforts and results have been presented with seemingly contradictory interpretations that have come under debate. For example, recent studies from this laboratory revealed that stainable bone aluminum and not total bone aluminum content is associated with the histologic bone abnormalities characteristic of aluminum-related bone disease (5), whereas Quarles et al. found that experimental osteomalacia in vitamin D-deficient dogs could be healed independently of presence or absence of stainable bone aluminum (6)-a finding interpreted as evidence that stainable bone aluminum represents an epiphenomenon of the osteomalacic state. Hodsman et al. postulate that the accumulation of osteoid in the skeleton represents a prerequisite for the accumulation of aluminum in bone (7).

This work was presented in part at the 18th annual meeting of the American Society of Nephrology, December 1985, New Orleans, LA.

Address reprint requests to Hartmut H. Malluche, M.D., Professor of Medicine and Director, Division of Nephrology, Bone and Mineral Metabolism, University of Kentucky Medical Center, 800 Rose Street, Lexington, KY 40536-0084. 1986.

Received for publication 9 June 1986 and in revised form 18 September

J. Clin. Invest.

(C) The American Society for Clinical Investigation, Inc.

0021-9738/87/03/0754/08 \$1.00

Volume 79, March 1987, 754-761
We found patients with predominant hyperparathyroid bone disease to have less stainable bone aluminum than those with mixed uremic osteodystrophy or low turnover osteomalacia (8) whereas Alfrey et al. found that uremic rats with intact parathyroid glands preferentially deposit aluminum in bone whereas parathyroidectomized uremic rats have less bone aluminum (9). Importantly, Lewis-Finch et al. have shown that osteomalacia develops in normal rats given aluminum only if parathyroidectomy is performed (10).

One possible interpretation of these research findings is to view them as evidence that the parathyroid hormone exerts a protective action on bone, an action preventing aluminum from being deposited in bone at a site critical for the development of aluminum-related bone disease. However, this interpretation must be considered in light of the fact that patients with extremely elevated parathyroid hormone levels may also have higher endogenous levels of calcitriol and that parathyroidectomy is associated with lower levels of calcitriol (11). Therefore, it appears imperative to investigate the influence, direct or indirect, of calcitriol on the aluminum deposition in bone.

It should be noted that whenever effects of parathyroid hormone or calcitriol on bone are studied, one has to take into consideration the fact that alterations in the concentrations of one hormone result in changes in the other. Consequently, our laboratory established a model that allows control of these corresponding changes (12). This study reports the use of that model to evaluate the single and combined effects of parathyroid hormone and calcitriol on the development of bone aluminum accumulation in experimental dogs with renal bone disease.

\section{Methods}

Experimental animals and protocol. 38 adult female beagle dogs, 3-5 yr of age with a mean weight of $9.8 \pm 0.2 \mathrm{~kg}$ (range $7.3-11.3 \mathrm{~kg}$ ) were purchased from a USDA-licensed dealer (Warren Hobble Beagles, Leesburg, $\mathrm{OH})$. The dogs were divided into four experimental groups of eight dogs each and one control group of six dogs. In the experimental groups, various combinations in status of calcitriol and parathyroid hormone were produced by $5 / 6$ nephrectomy (13) and thyroparathyroidectomy (14) followed by administration of calcitriol (1.25) and/or parathyroid hormone (PTH) ${ }^{1}$ at the following combinations: $1.25+/ \mathrm{PTH}+, 1.25-/$ PTH-, 1.25+/PTH-, and 1.25-/PTH+. The control group was sham operated. All surgeries were done using general anesthesia. Dogs developing progressive azotemia $(n=8)$ were excluded from the study.

Success of the parathyroidectomy was ascertained by histologic evaluation of the tissue removed during surgery and by a fall in serum calcium of at least $2 \mathrm{mg} / \mathrm{dl}$ during the $3 \mathrm{~d}$ following surgery. L-thyroxine (Synthroid, Flint, $\mathrm{NJ}$ ) was given orally at a dose of $0.02 \mathrm{mg} / \mathrm{kg}$ daily to maintain the dogs euthyroid (15). Euthyroidism was documented by measurements of $T_{3}$ and $T_{4}$, using a radioimmunoassay (Magic $T_{3}$ and $T_{4}$, Corning Glass Works, Palo Alto, CA). Autopsies were performed in all dogs at the end of the study and the region of the larynx, the carotid

1. Abbreviation used in this paper: PTH, parathyroid hormone. 
sheath and the mediastinum were carefully examined for regrowth of parathyroid tissue or accessory glands.

Group 1.25+/PTH $+(n=6)$ received chronic infusions of parathyroid hormone and daily subcutaneous injections of calcitriol. 1-34 synthetic bovine parathyroid hormone (Bachem Inc., Torrance, CA) was continuously infused during $8 \mathrm{mo}$ at a dose of $1.0 \mathrm{U} / \mathrm{kg}$ body wt/h. This dose was found to be necessary for maintenance of normocalcemia (12). Alzet osmotic minipumps (model 2002, Alza Corp., Palo Alto, CA) were implanted subcutaneously and changed every $12 \mathrm{~d}$ using local anesthesia. Infection or other side effects were not observed. The pumps were reported to be capable of maintaining a constant infusion for $14 \mathrm{~d}$ at a rate of 0.5 $\mu \mathrm{l} / \mathrm{h}(16,17) .10,000 \mathrm{U}$ of $1-34$ parathyroid hormone equivalent to 1 $\mathrm{mg}$ were dissolved in a solution containing $50 \mathrm{ml}$ physiologic saline 50 $\mu \mathrm{l} \mathrm{N} \mathrm{HCl}$ and $1.24 \mathrm{ml} 2 \%$ albumin. $2 \mathrm{ml}$ of this suspension of parathyroid hormone were transferred into the Alzet minipumps. The dogs tolerated the long-term infusion very well and no immobilization or change in activity was observed. Crystalline calcitriol (kindly supplied by Dr. Milan Uskokovic, Hoffmann-La Roche, Inc., Nutley, NJ) was dissolved in ethanol-propylene glycol (1:1) and given by daily subcutaneous injections between 8 and 9 a.m. Doses were adjusted to maintain normocalcemia. The mean dose administered to Group $1.25+/ \mathrm{PTH}+$ was $7.0 \pm 0.5 \mathrm{ng} /$ $\mathrm{kg}$ body wt/d (range $6.0-7.5 \mathrm{ng} / \mathrm{kg}$ body wt/d). Group $1.25-/ \mathrm{PTH}-(n$ $=4$ ) received the two vehicle solutions only (i.e., no parathyroid hormone or calcitriol); calcium lactate was supplemented as needed to maintain serum calcium levels in the normal range. The mean dose of calcium supplementation was $2.6 \pm 0.3 \mathrm{~g}$ calcium lactate/d (range $2.0-2.9 \mathrm{~g} / \mathrm{d}$ ). Group $1.25+/$ PTH $-(n=8)$ was given calcitriol at a mean dose of $11.7 \pm 1.3 \mathrm{ng} / \mathrm{kg}$ body $w \mathrm{t} / \mathrm{d}$ (range $7.5-15 \mathrm{ng} / \mathrm{kg}$ body $\mathrm{wt} / \mathrm{d}$ ) and the vehicle used for parathyroid hormone infusion. Group 1.25-/PTH+ $(n=6)$ received daily subcutaneous injections of the vehicle ethanol-propylene glycol and chronic infusion of parathyroid hormone by subcutaneous osmotic minipumps. Parathyroid hormone was infused at the same dose and for the same duration as in group $1.25+/ \mathrm{PTH}+$. The control group $(n=6)$ received parathyroid hormone vehicle by Alzet minipumps and daily subcutaneous injections with ethanol-propylene glycol. All dogs were fed a complete and balanced diet following the guidelines of the National Research Council (Alpo, Allen Products Co., Inc., Allentown, PA, horse meat chunks and beef by-products). They were fed once daily between 1:00 and 2:00 p.m. The diet consisted of $0.35 \%$ calcium, $0.25 \%$ phosphate, $11 \%$ crude protein, $5 \%$ crude fat, $1.5 \%$ crude fiber, $78 \%$ moisture, and $3 \%$ ash. Symptomatic hypocalcemia occurring during the days after parathyroidectomy was treated with $10 \%$ calcium gluconate i.v. as needed. Calcium lactate was mixed into the food as oral calcium supplement.

The need for administration of calcium lactate was continuously reassessed by monitoring serum calcium levels. Access to water was ad libitum. Renal osteodystrophy was allowed to develop with the various combinations in status of parathyroid hormone and calcitriol for a total of $8 \mathrm{mo}$. Thereafter, double labeling of bone was done in all animals using tetracycline hydrochloride at a dose of $20 \mathrm{mg} / \mathrm{kg}$ body wt/d for 2 $\mathrm{d}$ followed by a labeling free interval of $12 \mathrm{~d}$ and a second administration of tetracycline for $2 \mathrm{~d}$. $2 \mathrm{~d}$ thereafter, bone samples were obtained in an alternating manner from a standardized site of the right or left posterior inferior ilium (18). After bone biopsies all dogs received daily injections (on 6 of $7 \mathrm{~d}$ ) of aluminum lactate for $18 \mathrm{~d}$ at a dose of $0.5 \mathrm{mg} / \mathrm{kg}$ body $\mathrm{wt} / \mathrm{d}$. The aluminum lactate was dissolved in saline. The $\mathrm{pH}$ was adjusted with $\mathrm{NaOH}$ to 7.2. On day 1 and 2 and day 13 and 14 of aluminum administration, dogs received calcein for the second labeling of bone at a dose of $15 \mathrm{mg} / \mathrm{kg}$ body wt/d. After $18 \mathrm{~d}$ of aluminum administration, dogs were sacrificed and bone samples were obtained from the contralateral posterior inferior iliac bone.

Biochemical determinations. Blood was drawn once monthly for determination of serum calcium, phosphorus, and creatinine. Calcitriol, parathyroid hormone, and aluminum levels in blood were measured at baseline, i.e., before nephrectomy, and before and after administration of aluminum. All these blood samples were obtained between 8 and 9 a.m. before injection of the daily doses of calcitriol or vehicle. In addition, the kinetics or circadian changes of blood levels of calcitriol were measured in dogs receiving aluminum. Six dogs with and three dogs without calcitriol injections were studied. Blood was drawn at baseline, i.e., before injection of calcitriol or vehicle, and 20 and $40 \mathrm{~min}, 2,6,12$, and $24 \mathrm{~h}$ after injection. Serum calcium was measured by atomic absorption spectrophotometry (model 5000, Perkin-Elmer Corp., Norwalk, CT). Serum phosphorus and serum creatinine were measured by standard laboratory techniques (autoanalyzer, Technicon Instruments Corp., Tarrytown, NY). Blood levels of calcitriol were measured with a modification of the method described previously (11). The technique involved lipid extraction from 2-ml serum samples using acetonitrile and disposable C-18 columns (Waters Associates, Milford, MA) (19). This was followed by Sephadex LH-20 liquid chromatography and automated high-pressure liquid chromatography (HPLC) with Silica column (Waters Associates). The HPLC fraction containing calcitriol was collected and measured in duplicate by competitive protein-binding assay. Serum levels of parathyroid hormone were measured by a radioimmunoassay recognizing intact parathyroid hormone and synthetic 1-34 parathyroid hormone (Dr. David Endres, Nichols Institute, San Juan Capistrano, CA) directly on serum, and standards were prepared in buffer with carrier protein (20). Because hypoparathyroid serum was not used for preparations of standards, nonspecific serum or "ligand-free media" effects are seen with this assay, which results in some measurable parathyroid hormone even after successful parathyroidectomy. Aluminum concentration in serum and bone were measured by flameless atomic absorption spectrophotometry as described previously $(21,22)$. Bone aluminum content was determined as follows. All glassware was washed with detergent, soaked in $0.5 \%$ nitric acid, and rinsed thoroughly with deionized water. Wet bone was prepared before acid digestion as described previously (22). Bone marrow was removed by washing with a jet of deionized water. After drying at room temperature, bone samples were ground in stainless mills; powdered bone was then digested with acid, and aliquots were analyzed by flameless atomic absorption spectrophotometry. All determinations were done in duplicate.

Bone histology. Bone samples were fixed in ethanol, dehydrated, and embedded in methylmethacrylate for mineralized bone histology. Serial undecalcified sections of 3- and 7- $\mu$ meter thickness were cut using a Reichert Jung microtome (model 1140, Reichert Scientific Co., Buffalo, NY). 3- $\mu$ meter thick sections were stained with the modified Goldner trichrome stain (23), which permits discrimination between mineralized and nonmineralized bone and gives excellent cellular detail (24). 7- $\mu$ meter thick unstained sections were prepared for phase contrast and fluorescent light microscopy. In addition, $7-\mu \mathrm{m}$ thick sections were stained with the aurin tricarboxylic acid stain for detection of aluminum (25). Static and dynamic parameters of bone structure, bone formation, and resorption were measured using the Osteoplan system (Carl Zeiss, Thornwood, NY) as previously described (26). The extent of stainable aluminum in bone was evaluated with the same system.

The following histomorphometric parameters were obtained: $(a)$ trabecular bone mass, i.e., percentage of total bone occupied by trabecular bone; $(b)$ lamellar osteoid volume, i.e., percentage of trabecular bone occupied by lamellar osteoid; $(c)$ woven osteoid volume, i.e., percentage of trabecular bone occupied by woven osteoid; $(d)$ lamellar osteoid surface, i.e., percentage of trabecular surface covered by lamellar osteoid; (e) woven osteoid surface, i.e., percentage of trabecular surface covered by woven osteoid; $(f)$ thickness of lamellar osteoid; $(g)$ osteoid-osteoblast interface, i.e., percentage of osteoid covered by osteoblasts; $(h)$ osteoblastic index, i.e., number of osteoblasts per $100 \mathrm{~mm}$ trabecular boundary length (only plump rectangular cells juxtaposed to bone and arranged in a palisadelike manner were included in the measurement); ( $i$ ) bone-osteoclast interface, i.e., percentage of trabecular surface covered by mono or multinucleated osteoclasts; $(j)$ osteoclastic index, i.e., number of osteoclasts per $100 \mathrm{~mm}$ trabecular boundary length; $(k)$ mineral apposition rate, i.e., mean distance between tetracycline labels $\times 0.73 / \mathrm{d}$ of labeling-free interval; $(l)$ double-labeled osteoid, i.e., percentage of osteoid seams exhibiting two distinctively separated labels; $(m)$ bone formation rate, tissue level-surface referent, i.e., volume of mineralized bone made per unit of total trabecular surface and per unit time; $(n)$ bone formation rate per osteoblast, i.e., area of mineralized new bone made per osteoblast during 


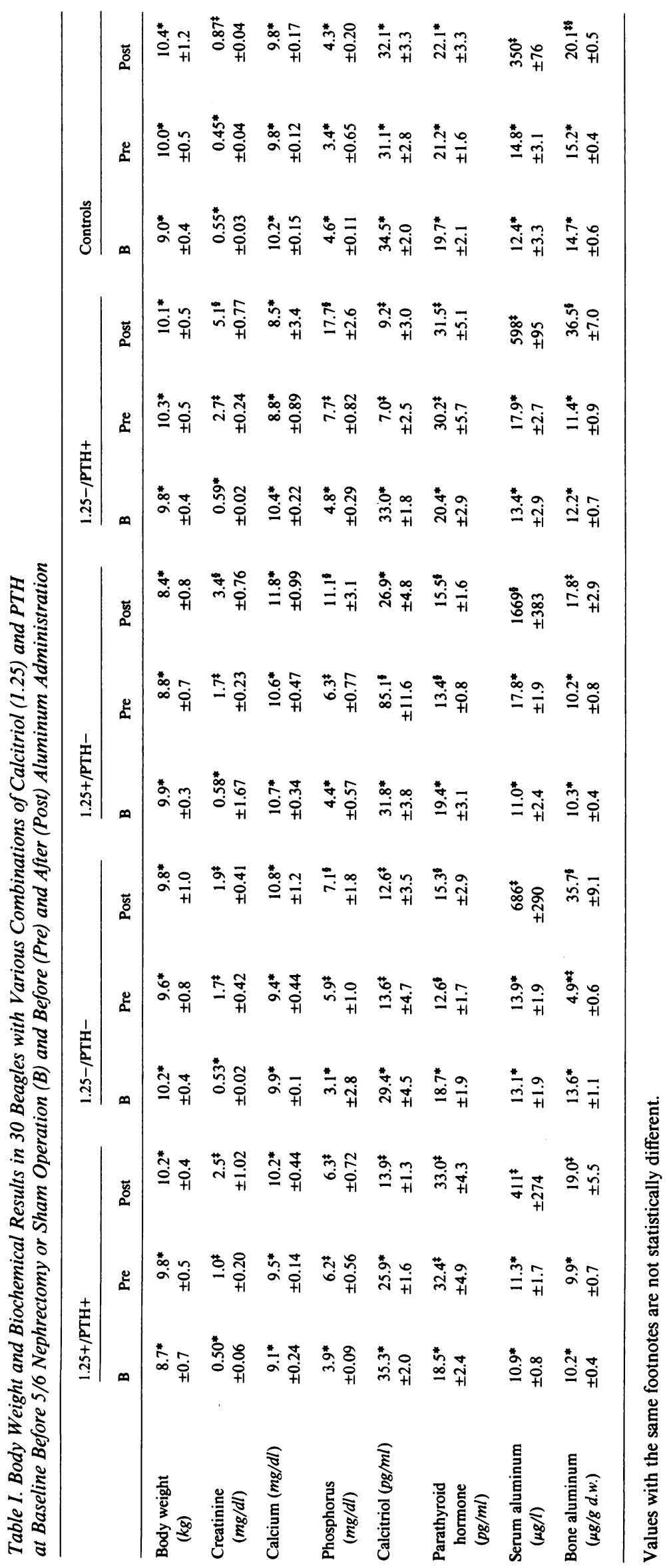


unit time; $(o)$ bone resorption rate per osteoclast, i.e., area of bone resorbed per osteoclast in unit time; $(p)$ stainable bone aluminum, i.e., fraction of trabecular surface exhibiting aluminum deposits at the bone-osteoid interface. At least fifty optical fields were evaluated at a magnification of $200 \times$ using an objective with 0.4 numerical aperture. All slides were read without knowledge of biochemical results or bone aluminum content.

Statistics. Statistical evaluation for differences between groups was done using unbalanced one-way analysis of variance; differences within groups were calculated using the Wilcoxon matched-pairs signed-rank test; correlations were calculated by regression analysis. All computations were performed with the SPSS ${ }^{+}$software package (SPSS, Inc., Chicago, IL) employing an IBM PC-AT computer. Results are given as mean values \pm standard error of the mean.

\section{Results}

Stable reduction in renal function with variation in status of calcitriol and parathyroid hormone was not associated with significant changes in body weight. Also, administration of aluminum for $18 \mathrm{~d}$ was not associated with significant changes in body weight. Serum biochemical results at baseline i.e., before nephrectomy, and before and after aluminum administration are shown in Table I. Serum creatinine before aluminum was moderately elevated compared with baseline in all dogs subjected to partial nephrectomy. Kidney function was comparable as documented by a lack of differences in serum creatinine between the groups at baseline as well as before aluminum administration. Kidney function fell after aluminum loading, however this fall did not reach statistical significance in dogs receiving both or no hormones (1.25+/PTH+, 1.25-/PTH-). Between baseline and before aluminum as well as before and after aluminum there were no differences in serum calcium levels between the groups. Serum phosphorus levels were higher than at baseline in the partially nephrectomized dogs before aluminum. They increased further after aluminum administration in groups with one hormone or no hormones. Serum concentrations of $T_{3}$ and $T_{4}$ were in the normal range in all dogs (27) and not different from baseline (baseline $\mathrm{T}_{3}: 78 \pm 7 \mathrm{ng} / \mathrm{dl} ; \mathrm{T}_{4}: 1.8 \pm 0.5 \mu \mathrm{g} / \mathrm{dl}$; before aluminum $\mathrm{T}_{3}: 72 \pm 6 \mathrm{ng} / \mathrm{dl} ; \mathrm{T}_{4}: 1.6 \pm 0.5 \mu \mathrm{g} / \mathrm{dl} ;$ after aluminum $\mathrm{T}_{3}$ : $\left.75 \pm 9 \mathrm{ng} / \mathrm{dl} ; \mathrm{T}_{4}: 1.9 \pm 0.6 \mu \mathrm{g} / \mathrm{dl}\right)$.
Surgical reduction of kidney function was associated with a fall in the endogenous production of calcitriol as evidenced by lower calcitriol levels before aluminum in nephrectomized dogs without calcitriol supplementation. Subcutaneous injections of calcitriol resulted in rises in blood levels of calcitriol with the highest levels measured $2 \mathrm{~h}$ after injection (Fig. 1). Blood levels fell at $12 \mathrm{~h}$ and returned to baseline $24 \mathrm{~h}$ after injection. No significant diurnal changes in serum levels of calcitriol were seen in five of six nephrectomized dogs without calcitriol administration (Fig. 1).

Administration of aluminum resulted in a fall in serum calcitriol levels in the dogs with calcitriol infusion. There were no significant changes in controls and no changes in dogs without calcitriol supplementation. Before aluminum administration, animals infused with parathyroid hormone had significantly higher levels of circulating parathyroid hormone compared with baseline and dogs without parathyroid hormone infusions had lower blood levels of the hormone. After aluminum, no significant changes in parathyroid hormone were observed in all groups. At autopsy no regrowth of parathyroid tissue or accessory glands were found.

Serum aluminum levels before aluminum administration in the experimental groups were not different from baseline. After aluminum administration, serum levels rose in all dogs. This increase was more pronounced in dogs receiving only calcitriol.

Administration of aluminum produced different degrees of stainable aluminum and different concentrations of aluminum in bone in the various experimental groups (Figs. 2 and 3, Tables I and II). The extent of stainable bone aluminum was significantly greater in the experimental groups with calcitriol deficiency. Absence or presence of parathyroid hormone did not affect stainable aluminum at the mineralization front. Extent of stainable bone aluminum in control animals was not significantly different from the animals receiving both calcitriol and parathyroid hormone. The group receiving calcitriol alone had even less stainable aluminum than controls $(P<0.05)$.

Measurements of bone aluminum content showed a similar pattern (Fig. 3). Animals with calcitriol deficiency had significantly higher bone aluminum concentrations than controls or

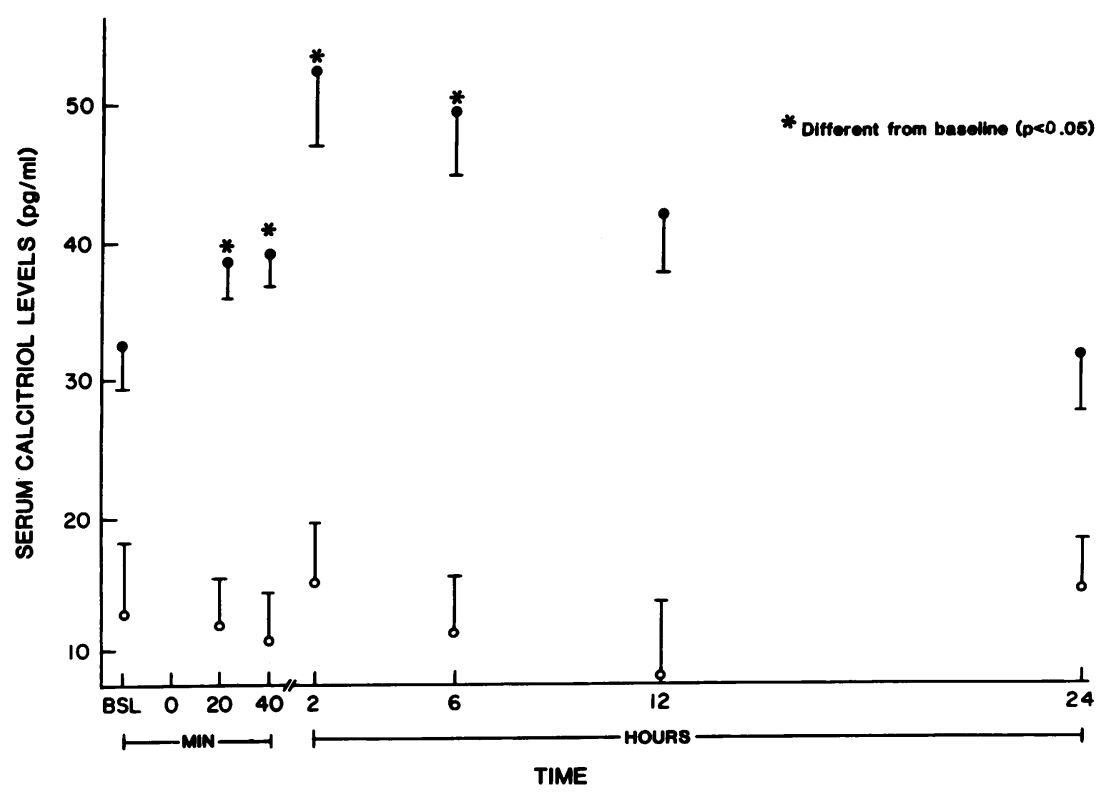

Figure 1. Serum concentrations of calcitriol during $24 \mathrm{~h}$ following subcutaneous administration of calcitriol or vehicle in $5 / 6$ nephrectomized beagle dogs receiving aluminum injections. (Solid circle) levels in dogs receiving calcitriol (dose, $7.9 \pm 1.4 \mathrm{ng} / \mathrm{kg}$. body wt; $n=6$ ); (open circle) levels in dogs receiving vehicle $(n=3)$. BSL, serum concentrations at baseline drawn 5-10 min before injections. 


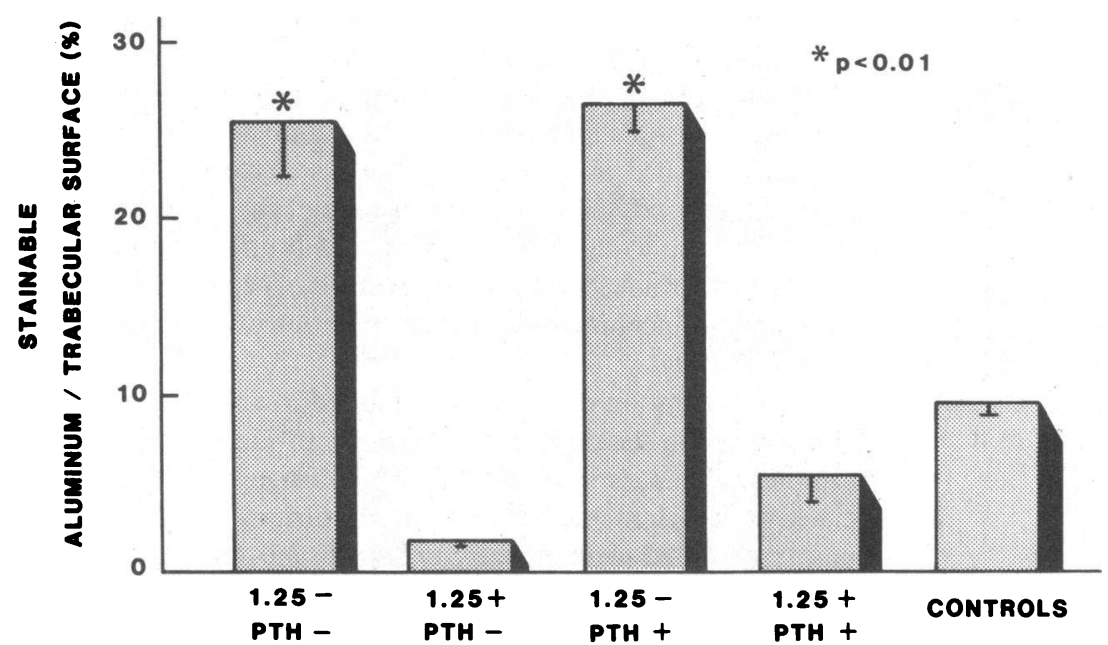

Figure 2. Percentage of trabecular surface with stainable aluminum at the bone-osteoid interface in $\%$ nephrectomized or sham-operated beagle dogs with various combinations in status of calcitriol (1.25) and PTH and in controls. those with calcitriol supplementation. Again, presence or absence of parathyroid hormone did not affect bone aluminum content in calcitriol-deficient dogs nor did it affect the bone aluminum content in calcitriol-replete dogs.

There was a linear correlation between stainable aluminum and bone aluminum content $(r=0.59, P<0.01)$. Stainable aluminum was inversely correlated with serum calcitriol (Fig. $4 ; r=-0.64, P<0.01$ ) and serum calcitriol correlated negatively with serum creatinine $(r=-0.50, P<0.01)$. No correlations were seen between stainable bone aluminum and serum creatinine and no correlations were found between serum parathyroid hormone and stainable bone aluminum, bone aluminum content, or serum creatinine.

Bone histology after surgical reduction of kidney function and induction of the various combinations in status of parathyroid hormone and calcitriol revealed changes similar to those reported recently by our group (12). Presence of parathyroid hormone was required for normal number of cells, whereas calcitriol was needed for normal cellular activity. Administration of aluminum to the dogs with different combinations in status of parathyroid hormone and calcitriol produced histomorphometric changes shown in Table II. Trabecular bone mass was not significantly altered. Lamellar osteoid volume increased in dogs with administration of one hormone only and woven osteoid volume decreased in all experimental groups. The same trend was observed for surface of lamellar and woven osteoid. Thickness of lamellar osteoid was increased in dogs with administration of one hormone only. Osteoid-osteoblast interface fell in all groups and osteoblastic index was significantly decreased in all dogs except those with very low numbers of osteoblasts before administration of aluminum, that is, in dogs without supplementation of both hormones (1.25-/PTH-). Changes in bone-osteoclast interface and number of osteoclasts were analogous to those observed for osteoblasts; that is, all groups with normal or high values before aluminum administration showed a significant reduction. Dynamic parameters of bone formation and resorption fell so dramatically that no double labeling of bone could be seen after the administration of aluminum in all experimental groups. In the controls there was a significant fall in mineral apposition rate, double-labeled osteoid and bone formation rates at the tissue and cellular level. Bone resorption rates fell accordingly.

\section{Discussion}

The results of our study confirm that aluminum causes decreased bon'e formation and impairs mineralization as evidenced by nonmeasurable or low bone formation and mineral apposition

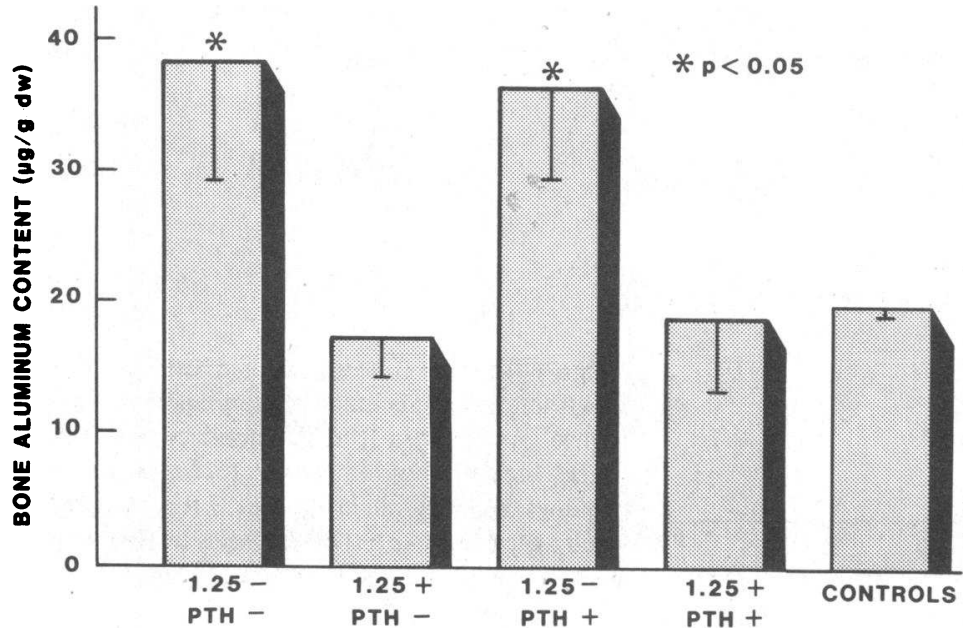

Figure 3. Bone aluminum content in $5 / 6$ nephrectomized or sham-operated beagle dogs with various combinations in status of calcitriol (1.25) and PTH and in controls. 


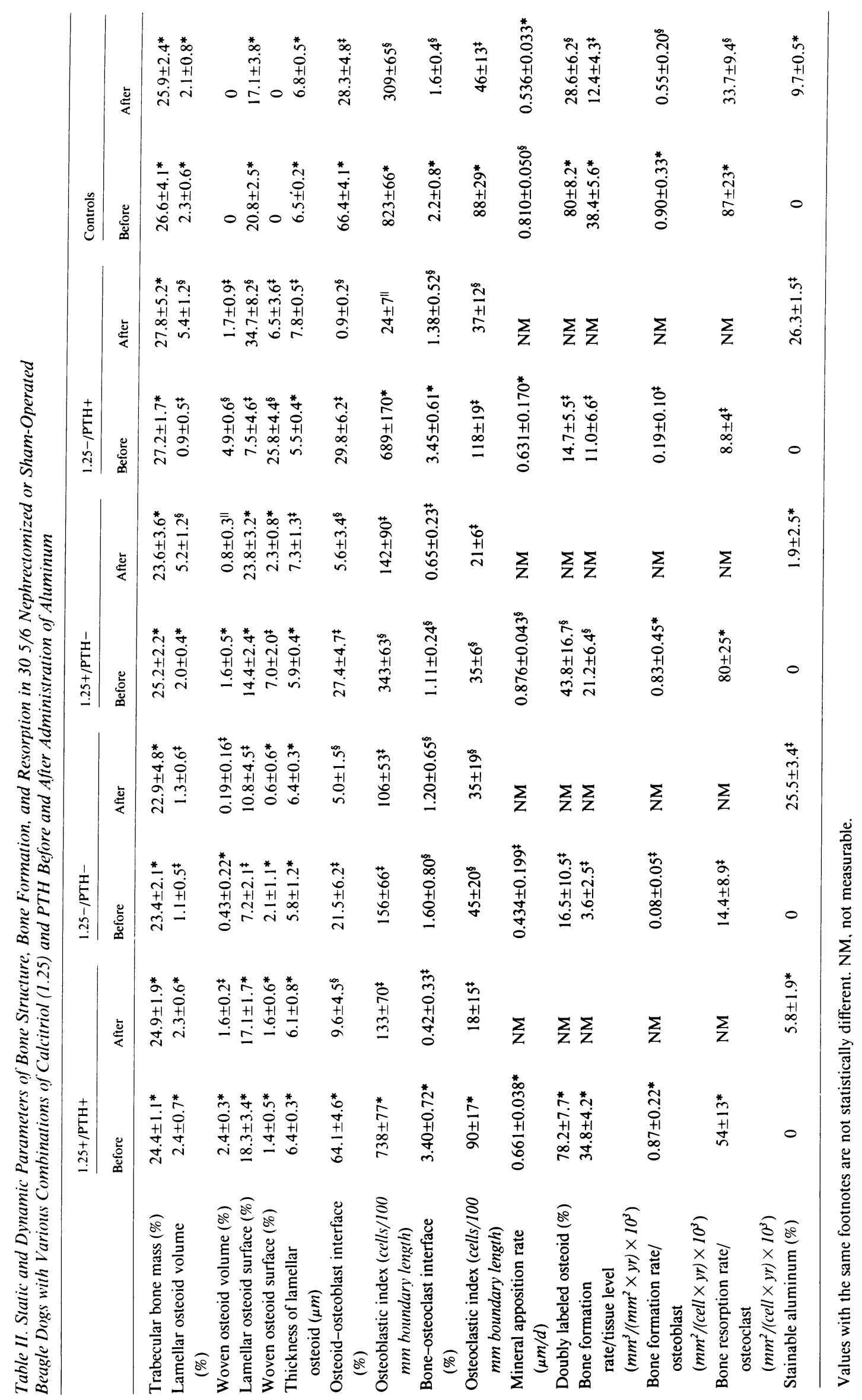




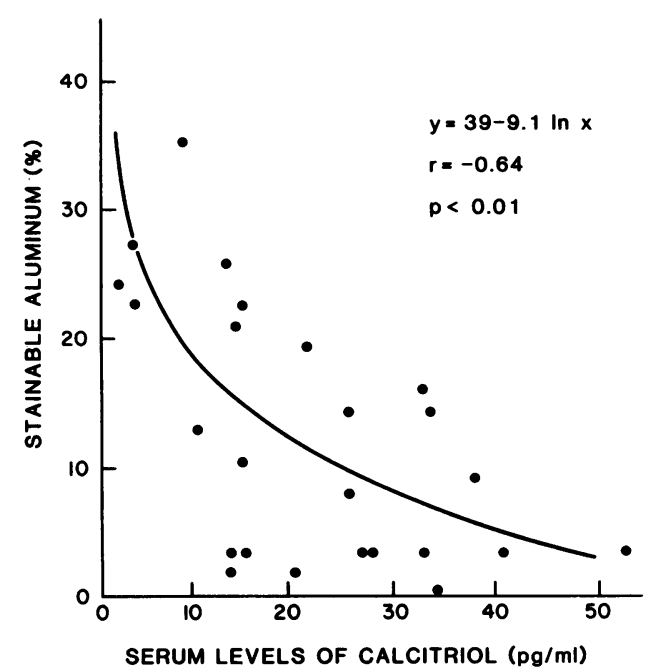

Figure 4. Relationship between serum levels of calcitriol and the percentage of trabecular surface with stainable aluminum at the bone-osteoid interface in $5 / 6$ nephrectomized beagle dogs after aluminum administration.

rates in the experimental dogs as well as in the control dogs treated with aluminum. Furthermore, the differences in bone histology observed before and after aluminum administration are similar to those seen in cross-sectional studies comparing uremic patients with and without aluminum-related bone abnormalities $(2,3,8)$.

Not only does this study extend the previous research observations in dogs and rats $(7,28-30)$, it also reports the novel finding of higher stainable bone aluminum and higher bone aluminum content in calcitriol-deficient dogs, thus ascribing a role in the development of aluminum accumulation in bone to calcitriol. While this finding seems to dispute the notion that hypoparathyroidism plays a causative role in the genesis of aluminum-induced osteomalacia $(9,10)$, the discrepancy may be attributable to the following. (a) The latter studies were conducted on rats that are continuously growing, and therefore the reported changes might not take place in adult dogs whose skeletons are much more akin to that of the human. (b) The rats given aluminum did not have preexisting uremic bone disease, and the aluminum was administered over a much longer period of time. There is a strong possibility that the parathyroidectomies performed on the experimental rats caused lower circulating levels of calcitriol that, in turn, may well have affected bone aluminum uptake. Indeed, we found a fall in endogenous levels of calcitriol after parathyroidectomy in chronically dialyzed patients (11). The results of this study are in keeping with the clinical observation that stainable bone aluminum is less prevalent in patients with predominant hyperparathyroid bone disease than in those with low turnover osteomalacia $(1,8)$. The results are also in agreement with the finding that parathyroidectomy is associated with higher aluminum accretion rates in bone (31).

Studies conducted on rats by Hodsman et al., revealed higher bone aluminum content in vitamin D-depleted rats compared to vitamin D-replete rats (7) and led the authors to conclude that accumulation of osteoid in vitamin D deficiency represents the factor responsible for aluminum accumulation. The results of our study of accumulation of aluminum in the bone of dogs with and without preexisting osteoid accumulation argue against the conclusion. Only future studies will determine whether our findings of more pronounced changes in surface and volume of lamellar osteoid in aluminum-treated dogs with one hormone reflect the imbalance caused by the administration of the hormone, or whether these changes are related to other factors. It should be noted, however, that the inverse correlation observed between calcitriol and stainable bone aluminum and the lack of correlation between parathyroid hormone and stainable bone aluminum strongly suggest that calcitriol deficiency and not hypoparathyroidism plays the dominant role in the accumulation of aluminum in bone.

Another result of this study indicates that serum levels of calcitriol also correlated inversely, though somewhat weaker, with serum creatinine. However, because serum creatinine did not correlate with stainable bone aluminum, our results indicate that the differences in stainable aluminum between the experimental groups are not due to differences in renal function. Further studies are necessary to determine whether the lower aluminum accumulation in bone of calcitriol-replete dogs is accompanied with higher aluminum content in other organs. Hirschberg et al. have shown that calcitriol and parathyroid hormone may affect the distribution of aluminum in organs of experimental rats (32). They find that calcitriol enhances the uptake of aluminum in the heart and muscles, yet they also reveal decreased aluminum content in the bone, liver, and brain of animals receiving calcitriol and parathyroid hormone. Differing organ distribution of aluminum was also demonstrated by Alfrey et al. in uremic and normal rats (9). Quarles et al. have shown that the accumulation of stainable aluminum at the mineralization front of vitamin D-deficient dogs can be reversed by the administration of calcitriol-a reversal that occurs despite continued aluminum injections (6). These observations are in keeping with our findings of lower stainable bone aluminum in calcitriol-replete dogs than in calcitriol deficient ones.

It is important to emphasize that our findings should not be interpreted as justification for calcitriol therapy in cases of advanced aluminum-related bone disease. Patients with severe aluminum-related bone disease and stainable bone aluminum at $>70 \%$ of the trabecular surface are known to become readily hypercalcemic when treated with calcitriol. This complication prohibits a therapeutic regimen using calcitriol for such patients. Further studies will determine whether maintenance of normal or high circulating levels of calcitriol in dialysis patients without bone aluminum or with mild-to-moderate aluminum accumulation will prevent progressive deposition of aluminum in bone and the development of aluminum-related bone disease.

\section{Acknowledgments}

We are indebted to Howard Dahlman and Richard Wheaton for skillful technical assistance. We thank Kim Baize and Pat Scott for secretarial assistance.

This work was supported in part by the National Institutes of Health grant 1R01-AM34419 and Dialysis Clinics Inc. grant \#90, Lexington, $\mathrm{KY}$.

\section{References}

1. Cournot-Witmer, G., J. Zingraff, J. J. Plachot, F. Escaig, R. Lefevre, P. Boumati, A. Bordeau, M. Garabedian, P. Galle, R. Bourdon, T. Drueke, and S. Balsan. 1981. Aluminum localization in bone from hemodialyzed patients: relationship to matrix mineralization. Kidney Int. 20:375-385. 
2. Ott, S. M., N. A. Maloney, J. W. Coburn, and A. C. Alfrey. 1982. The prevalence of aluminum in renal osteodystrophy and its relationship to response to calcitriol therapy. N. Engl. J. Med. 307:709-713.

3. Hodsman, A. B., D. J. Sherrard, A. C. Alfrey, S. M. Ott, A. S. Brickman, A. L. Miller, N. A. Maloney, and J. W. Coburn. 1982. Bone aluminum and histomorphometric features of renal osteodystrophy. $J$. Clin. Endocrinol. Metab. 54:539-546.

4. Smith, A. J., M. C. Faugere, K. Abreo, P. Fanti, B. Julian, and H. H. Malluche. 1986. Aluminum related bone disease in mild and advanced renal failure. Evidence for high prevalence and morbidity, and studies on etiology and diagnosis in 197 patients. Am. J. Nephrol. 6: 275-283.

5. Faugere, M. C., and H. H. Malluche. 1986. Stainable aluminum and not aluminum content reflects histologic changes in bone of dialyzed patients. Kidney Int. 30:717-722.

6. Quarles, L. D., V. W. Dennis, H. J. Gitelman, J. Harrelson, and M. K. Drezner. 1985. Aluminum deposition at the osteoid-bone interface. An epiphenomenon of the osteomalacia state in vitamin D-deficient dogs. J. Clin. Invest. 75:1441-1447.

7. Hodsman, A. B., C. Anderson, and F. Y. Leung. 1984. Accelerated accumulation of aluminum by osteoid matrix in vitamin $\mathrm{D}$ deficiency. Miner. Electrolyte Metab. 10:309-315.

8. Malluche, H. H., and M. C. Faugere. 1985. Aluminum: toxin or innocent bystander in renal osteodystrophy. Am. J. Kidney Dis. 6:336341.

9. Alfrey, A. C., A. Sedman, and L. Chany. 1985. The compartmentalization and metabolism of aluminum in uremic rats. J. Lab. Clin. Med. 105:227-233.

10. Lewis-Finch, J., M. Bergfeld, K. Martin, S. Teitelbaum, and E. Slatopolsky. 1986. Hypoparathyroidism is essential for development of aluminum-induced osteomalacia in rats. Kidney Int. 29:218. (Abstr.)

11. Fanti, P., A. J. Smith, P. A. Price, R. E. Reitz, and H. H. Malluche. 1986. Effects of parathyroidectomy on circulating levels of $1 \alpha, 25$-dihydroxyvitamin D and bone Gla protein in dialyzed patients. J. Clin. Endocrinol. Metab. 62:869-873.

12. Malluche, H. H., C. Matthews, M. C. Faugere, P. Fanti, D. B. Endres, and R. M. Friedler. 1986. 1,25(OH $)_{2} \mathrm{D}_{3}$ maintains bone cell activity and parathyroid hormone modulates bone cell number in experimental dogs. Endocrinology. 119:1298-1304.

13. Platt, R., M. H. Roscoe, and F. W. Smith. 1952. Experimental renal failure. Clin. Sci. (Lond.). ii:217-231.

14. Friedler, R. M., K. Kurokawa, J. W. Coburn, and S. G. Massry. 1975. Renal action of Cholera toxin I. Effects on urinary excretion of electrolytes and cyclic AMP. Kidney Int. 7:77-85.

15. Hightower, D., J. R. Kyzar, D. K. Chester, and E. M. Wright. 1973. Replacement therapy for induced hypothyroidism in dogs. J. Am. Vet. Med. Assoc. 163:979-980.

16. Obie, J. F., and C. W. Cooper. 1979. Loss of calcemic effects on calcitonin and parathyroid hormone infused continuously into rats using the Alzet Osmotic Minipump. J. Pharmacol. Exp. Ther. 209:422-428.
17. Trechsel, V., J. A. Elsman, J. A. Fischer, J. P. Bonjour, and H. Fleisch. 1980. Calcium-dependent parathyroid hormone-independent regulation of 1,25-dihydroxyvitamin D. Am. J. Physiol. 239:E119-E124.

18. Hardt, A. B., and W. S. S. Jee. 1982. Trabecular bone structural variation in biopsy sites of the beagle ilium. Calcif. Tissue Int. 34:391395.

19. Reinhardt, T. A., R. L. Horst, J. W. Orf, and B. W. Hollis. 1984. A microassay for 1,25-dihydroxyvitamin $\mathrm{D}$ not requiring high performance liquid chromatography: application to clinical studies. J. Clin. Endocrinol. Metab. 58:91-98.

20. Segre, G. V. 1983. Amino-terminal radioimmunoassay for human parathyroid hormone. Clin. Disorders Bone Miner. Metab. 617:14-17.

21. Legendre, G. R., and A. C. Alfrey. 1976. Measuring picogram amounts of aluminum in biological tissue by flameless atomic absorption analysis of a chelate. Clin. Chem. 22:53-56.

22. Freundlich, M., G. Zilleruelo, C. Abitbol, J. Strauss, M. C. Faugere, and H. H. Malluche. 1985. Infant formula as a cause of aluminum toxicity in neonatal uraemia. Lancet. ii:527-529.

23. Goldner, J. 1938. A modification of the Masson trichrome technique for routine laboratory purposes. Am. J. Pathol. 14:237-242.

24. Malluche, H. H., W. Meyer, D. Sherman, and S. G. Massry. 1982. Quantitative bone histology in 84 normal American subjects. Micromorphometric analysis and evaluation of variance of iliac crest bone. Calcif. Tissue Int. 34:449-455.

25. Lillie, P. D., and H. M. Fullmer. 1976. Histopathologic technique and practical histochemistry. Fourth edition. McGraw-Hill Book Co., New York.

26. Malluche, H. H., D. Sherman, W. Meyer, R. Manaka, and S. C. Massry. 1982. A new semiautomatic method for quantitative static and dynamic bone histology. Calcif. Tissue Int. 34:439-448.

27. Doering, G. G., and L. A. Corwin. 1980. Clinical hypothyroidism correlated with $\mathrm{T}_{3}$ and $\mathrm{T}_{4}$ tests. J. Am. Vet. Med. Assoc. 163:1186.

28. Ellis, H. A., J. H. McCarthy, and J. Herrington. 1979. Bone aluminum in hemodialyzed patients and in rats injected with aluminum chloride: relationship to impaired bone mineralization. J. Clin. Pathol. (Lond.). 32:832-844.

29. Goodman, W. F., D. A. Henry, R. Horst, R. K. Nudelman, A. A. Alfrey, and J. W. Coburn. 1984. Parenteral aluminum administration in the dogs. II. Induction of osteomalacia and effect on vitamin D metabolism. Kidney Int. 25:370-375.

30. Chan, Y., A. C. Alfrey, and S. Posen. 1983. The effect of aluminum on normal and uremic rats. Tissue distribution, vitamin $D$ metabolites and quantitative bone histology. Calcif. Tissue Int. 35:344-351.

31. Andress, D. L., S. M. Ott, N. A. Maloney, and D. J. Sherrard. 1985. Effect of parathyroidectomy on bone aluminum accumulation in chronic renal failure. $N$. Engl. J. Med. 312:468-472.

32. Hirschberg, R., D. von Herrath, R. Voss, W. Bossaler, U. Mauelshagen, A. Pauls, and K. Schaffer. 1985. Organ distribution of aluminum in uremic rats: influence of parathyroid hormone and 1.25 dihydroxyvitamin $\mathrm{D}_{3}$. Miner. Electrolyte Metab. 11:106-110. 